\title{
Intrinsic Brain Network Biomarkers of Antidepressant Response: a Review
}

\author{
Katharine Dunlop ${ }^{1} \cdot$ Aleksandr Talishinsky $^{1} \cdot$ Conor Liston $^{1,2}$
}

Published online: 13 August 2019

(C) The Author(s) 2019

\begin{abstract}
Purpose of Review Poor treatment response is a hallmark of major depressive disorder. To tackle this problem, recent neuroimaging studies have sought to characterize antidepressant response in terms of pretreatment differences in intrinsic functional brain networks. Our aim is to review recent studies that predict antidepressant response using intrinsic network connectivity. We discuss current methodological limitations and directions for future antidepressant biomarker studies.

Recent Findings Functional connectivity stemming from the subgenual and rostral anterior cingulate has shown particular consistency in predicting antidepressant response. Differences in this connectivity may prove fruitful in differentiating treatment responders to many antidepressant interventions. Future biomarker studies should integrate biological MDD subtypes to address the disorder's inherent clinical heterogeneity.

Summary These clinical and scientific advancements have the potential to address this population marked by limited treatment response. Methodological considerations, including patient selection, response criteria, and model overfitting, will require future investigation to ensure that biomarkers generalize for prospective prediction of treatment response.
\end{abstract}

Keywords Major depressive disorder $\cdot$ Biomarker $\cdot$ Treatment response $\cdot$ Predictor $\cdot$ Brain networks $\cdot$ Network connectivity

\section{Introduction}

A hallmark feature of major depressive disorder (MDD) is patients' limited treatment responsivity to conventional pharmacotherapy and psychotherapy. As demonstrated by the seminal STAR $*$ D trial, approximately one third of patients remit to their first antidepressant, and a given patient's likelihood of remission diminishes with each successive monotherapy [1]. Those who do not respond to two or more antidepressant trials are considered to have treatment-resistant depression (TRD); TRD is estimated to affect 35\% of MDD patients [2]. These limited response rates may be related to the fact that

This article is part of the Topical Collection on Precision Medicine in Psychiatry

Katharine Dunlop

kad2032@med.cornell.edu

1 Brain and Mind Research Institute, Weill Cornell Medicine, 413 East 69th Street, Box 240, New York, NY 10021, USA

2 Department of Psychiatry, Weill Cornell Medicine, New York, NY, USA
MDD is a highly heterogeneous disorder [3••], and individual patients may subsequently require tailored treatments.

Motivated by these poor response rates, efforts have been made to identify pretreatment clinical characteristics that predict response to antidepressant interventions [4]. For example, comorbid psychiatric or somatic disorders are associated with poorer outcomes to pharmacotherapies. In the STAR*D trial, the remission rate of patients with severe somatic symptoms dropped to only $29.3 \%$ across three successive monotherapies [5]. It is important to note that although clinical factors such as somatic symptoms show significance at the group level, none have yet achieved the much higher bar of clinically meaningful predictive value at the individual level.

In light of these studies showing limited individual predictive value for clinical measures, recent studies have sought to advance our understanding of antidepressant response in terms of pretreatment differences in biological measures. Abnormal structural and functional connectivity within or between functional brain networks are present across a wide variety of psychiatric illnesses [6]. Promising results have been reported in a growing oeuvre of neuroimaging studies that have assessed the accuracy of MRI connectivity measures in predicting antidepressant response [7]. 
In this article, our aim is to review recent studies identifying intrinsic brain network differences that predict antidepressant response. First, we provide a brief background on functional brain networks and the specific networks pertinent to MDD. Next, we review recent studies reporting pretreatment differences in brain network activity or connectivity that characterize treatment response. Finally, we discuss current clinical challenges and methodological drawbacks, and comment on areas of improvement for the next generation of antidepressant biomarker studies.

\section{Intrinsic Brain Networks and Their Relationship to MDD}

\section{What Are Intrinsic Brain Networks?}

Over the past two decades, functional neuroimaging studies have consistently found that the brain exhibits spontaneous, low-frequency fluctuations in the fMRI BOLD signal and that interregional correlations in these fluctuations can be used to define large-scale networks that are anatomically and functionally distinct [8]. These networks are discernible from both ongoing spontaneous fluctuations in brain activity [9] and from neuroimaging harnessing cognitive or behavioral paradigms [10]. Consequently, we term these networks intrinsic brain networks (IBNs): distinct networks of functionally coupled brain regions whose spontaneous or task-evoked fluctuations in activity are correlated over time [9]. The spatial motifs of IBNs are highly replicable, with strong intraindividual [11] and interindividual consistency [12].

Large-scale IBNs are also hypothesized to reflect the underlying structural topology of the brain [13, 14], and connectivity within or between IBNs has been associated with human cognition and behavior through a variety of paradigms, including cognitive control tasks [15], motor and language performance [16], impulsivity [17], and states of consciousness [18]. While not yet fully understood, current studies suggest that IBNs operate in concert to facilitate complex cognitive and behavioral processes [19].

\section{Functional Networks Pertinent to MDD Symptomatology}

While the exact number of IBNs is not yet fully known, most human neuroimaging studies report the existence of 7-17 IBNs $[19,20]$. Furthermore, studies of structural and functional MRI, electroencephalography, and positron emission tomography have identified at least four candidate IBNs involved in MDD pathophysiology: the default mode network $(\mathrm{DMN})$, salience network (SN), central executive network $(\mathrm{CEN})$, ventromedial affect network (VMN), and IBNs related to autonomic and limbic function (reviewed extensively in
[21, 22]) (Fig. 1a, b). To summarize, negative self-referential rumination is associated with disrupted connectivity of the SN and CEN with the DMN and VMN, as well as hyperactivation stemming from the subgenual cingulate cortex $[25,26]$. Similarly, MDD patients exhibit DMN and VMN hyperactivity to aversive or nonrewarding stimuli [27-29]. Conversely, hypoactivity of attentional and ventromedial reward networks (SN and VMN) has been linked to poor incentive salience and anhedonia, another hallmark MDD symptom [30-32]. MDD patients also display deficits in cognitive control compared to nondepressed controls, and this deficiency has been attributed to abnormal functioning of the SN and CEN [33-36].

\section{Brain Network Biomarkers of Antidepressant Response}

\section{Default Mode Network}

The DMN is comprised of the posterior cingulate cortex (PCC), precuneus, medial prefrontal cortex (mPFC), rostral anterior cingulate cortex (rACC), bilateral inferior parietal cortex (IPL), and medial/lateral temporal cortices [37] (Fig. 1a). Recent studies have reported that DMN activation is related to a number of behaviors related to internally generated cognition and self-referential processing [38], including mind wandering [39], autobiographical memory retrieval [40], spatial navigation, and theory of mind [41]. Furthermore, the DMN appears to consist of at least three subnetworks: a midline "core" network comprised of the MPFC and PCC that is consistently activated for all DMN-relevant functions; a dorsal mPFC subnetwork that co-activates with the angular gyrus and temporal pole for self-referential or affective processes; and a temporoparietal network comprised of the IPL, temporal cortices for memory retrieval and scene reconstruction [20, 42].

Recent studies frequently report that volumetric decreases and functional activity of the midline "core" subnetwork predict antidepressant response. Anteriorly, larger baseline $\mathrm{rACC} / \mathrm{mPFC}$ volume is significantly associated with response to fluoxetine [43], chronotherapeutics [44], and internet-based cognitive therapy [45]. In older adults, rACC volume [46] and white mater integrity is positively correlated with escitalopram response and improvements in negative self-referential rumination [47]. However, pretreatment rACC volume is not significantly associated with response to all pharmacotherapy. For sertraline response, rACC volume increases within the first week of treatment and is significantly correlated with improvement at 8 weeks; however, these structural indices individually predicted escitalopram response with an accuracy of only $65 \%[48 \bullet$. Functionally, resting-state rACC theta has been correlated with antidepressant response in two large pharmacotherapy trials with conflicting results: one study 

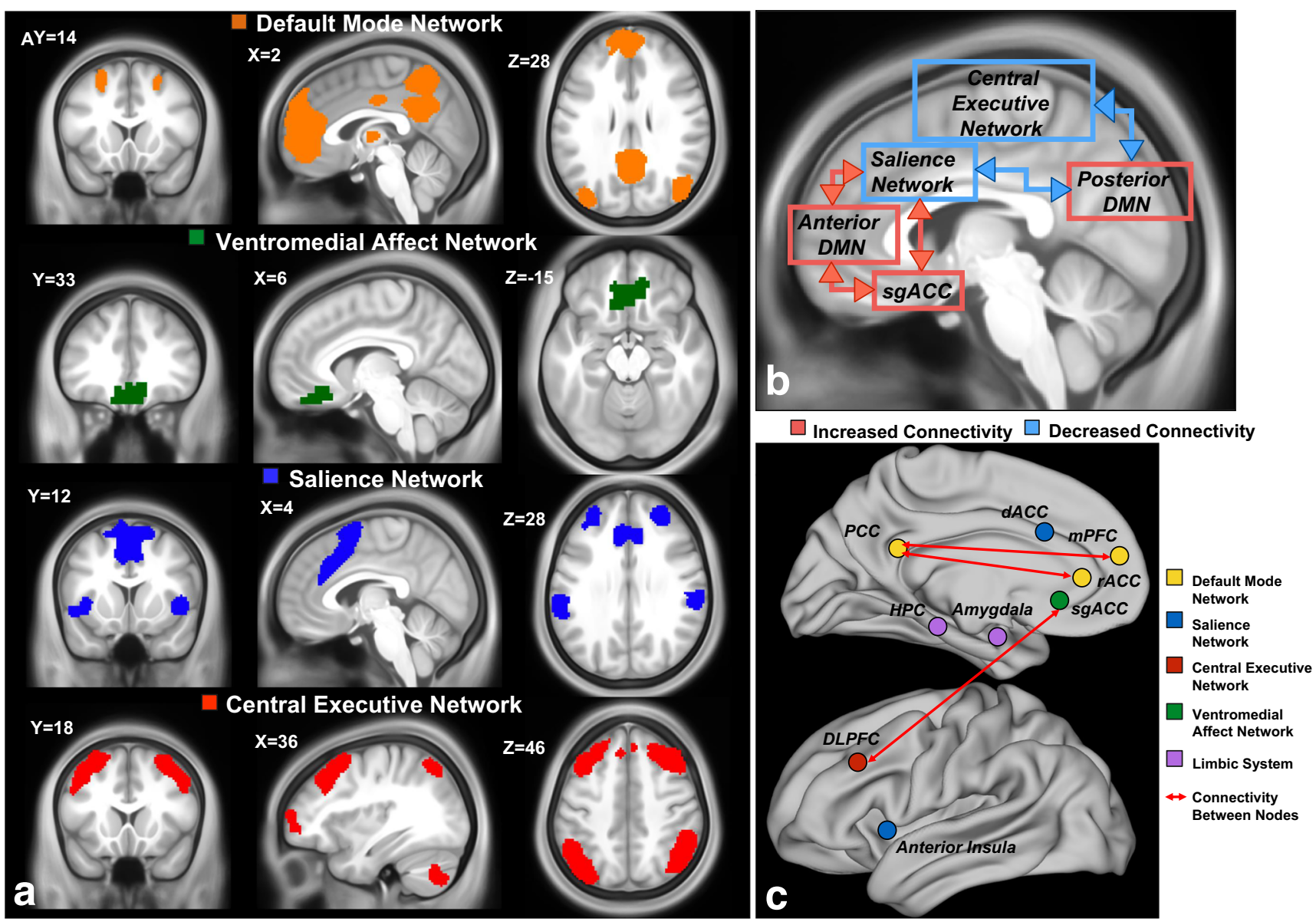

Fig. 1 Intrinsic brain networks may be used to differentiate depressed treatment responders from nonresponders. a The four intrinsic brain networks implicated in MDD (default mode $=$ yellow, salience $=$ blue, central executive $=$ red, and ventromedial reward $=$ green $)$. Networks were generated using [23]. b Connectivity changes commonly reproduced in depressed patients compared to healthy controls (adapted

reported that high frontal theta was associated with nonresponse [49], while the other showed the converse [50••].

Posterior nodes of the midline subnetwork have also been shown to correlate with antidepressant response. For example, larger baseline PCC volume is correlated with pharmacotherapy response [51], and higher pretreatment glucose metabolism in the precuneus is linked to psychotherapy completion [52]. Additionally, deactivation of both the precuneus and PCC during an emotion discrimination task correlated with improvements after 2 weeks of pharmacotherapy, but not after 4 weeks [53].

Connectivity between the anterior and posterior regions of the DMN also correlates with antidepressant response. Five recent studies have reported that stronger baseline posterior DMN (PCC) connectivity to the anterior DMN (mPFC) correlates with antidepressant response to electroconvulsive therapy (ECT) [54] and pharmacotherapy [55-58]. Furthermore, four of these studies were able to correctly classify participants as responders with $>80 \%$ accuracy $[54,55,57,58]$. from [24]). Red boxes and lines indicate increased within-network and between-network connectivity, respectively. Blue boxes and lines indicate decreased within-network and between-network connectivity, respectively. c Nodes and connectivity implicated in predicting antidepressant treatment response, colored by intrinsic brain network membership

\section{Ventromedial Affect and Reward Networks}

Affective and reward networks have long been implicated in the pathophysiology of depression: the subgenual anterior cingulate cortex (sgACC), a central node involved in affect, was one of the earliest neuroimaging biomarkers of depression and antidepressant response [28, 59-63]. Since then, an affectassociated network with strong connectivity to the sgACC has been repeatedly implicated in depressive affect and treatment response [30, 64]. In this review, we broadly define the $\mathrm{VMN}$ as encompassing the sgACC, nucleus accumbens (NAcc), medial OFC (mOFC), and ventromedial prefrontal cortex (VMPFC) [65] (Fig. 1a).

Within the past decade, neuroimaging has provided several lines of evidence supporting a role of the sgACC in predicting antidepressant response. Importantly, variations in sgACC structure, function, and connectivity have been associated with response to many different interventions. These variations suggest that sgACC function may be used to guide 
treatment selection. For example, early PET studies reported that treatment response can be associated with both increased and decreased $\mathrm{sgACC}$ metabolism for noninvasive brain stimulation [66] and CBT combined with SSRI treatment, respectively $[67,68]$. In addition to baseline metabolic activity, structural MRI studies have shown that greater baseline sgACC volume correlated with clinical response to CBT [69] and ECT [70]. Some [71, 72] but not all [73] task-based fMRI studies have supported the notion that lower sgACC activity in response to emotional stimuli predicts response to CBT.

Similar to studies assessing structural, metabolic, and taskevoked biomarkers, resting-state fMRI studies have shown sgACC functional connectivity to predict treatment response to pharmacotherapy, psychotherapy, and TMS. Crucially, the spatial topography of sgACC functional connectivity may help to guide treatment selection. Studies investigating connectivity between sgACC and prefrontal regions, including the dorsomedial prefrontal cortex (DMPFC), dorsolateral prefrontal cortex (DLPFC), VMPFC, ventrolateral prefrontal cortex (VLPFC), and medial orbitofrontal cortex, have shown more positive baseline functional connectivity to be associated with improved treatment outcome for pharmacotherapy and psychotherapy [74, 75••, 76-78]. On the other hand, more negative baseline functional connectivity between sgACC and the ACC, DMN, and parts of the left superior MPFC has also been associated with improved treatment outcome for noninvasive brain stimulation targeting the DLPFC $[58,78]$.

\section{Salience and Attentional Networks}

The $\mathrm{SN}$ is predominantly comprised of the $\mathrm{dACC}$ and anterior insula (AI) [79] (Fig. 1a), and its activity is related to the detection of and autonomic reactions to salient stimuli [80]. $\mathrm{SN}$ activity is related to goal-directed behavior, meaning that the SN identifies relevant external information [81, 82] and projects to motor and premotor regions via the $\mathrm{dACC}$ to initiate a behavior $[83,84]$. AI and dACC activity is also positively correlated with affective face discrimination [85].

Four studies have reported that antidepressant response is associated with pretreatment dACC activity during emotion processing and affect regulation. Escitalopram response was associated with high baseline dACC activity to faces displaying negative versus positive affect [86], with a greater reduction in this contrast after the first week of treatment [87]. However, baseline dACC hyperactivity correctly classified escitalopram response at roughly $70 \%$ accuracy [86]. Baseline AACC hyperactivity during emotion processing has also significantly correlated to antidepressant response to chronotherapeutics [44] and venlafaxine [88] with comparable classification accuracy.

Insula activity during emotion processing has also been linked with antidepressant response, with hyperactivity during negative emotional images being predictive of response to mirtazapine, venlafaxine [89], and combination pharmacotherapy [90]. Similarly, a greater reduction in insular activity to negative facial expressions within the first week of escitalopram is predictive of later antidepressant response [87]. Also of note, insula hyper- or hypometabolism may discriminate between response to pharmacotherapy versus psychotherapy, with hyperactivity corresponding to escitalopram response and the opposite to cognitive behavioral therapy [91].

Notably, SN activity has been shown to predict antidepressant response in TRD to repetitive transcranial magnetic stimulation (rTMS) targeting both the DLPFC and DMPFC. DLPFC-rTMS responders display increased SN functional connectivity on resting-state fMRI [92] and theta power and connectivity using electroencephalography (EEG) [93]. Both of these studies report sensitivities and specificities $>80 \%$ using these measures. DMPFC-rTMS responders display higher baseline SN activity to the sgACC in one early openlabel study [74], highlighting the potential of noninvasive neurostimulatory targets to access networks that are modulated by invasive therapeutics like DBS [94].

\section{Frontoparietal Central Executive Network}

The DLPFC, frontal eye fields, and superior parietal cortex are the core nodes of the CEN. The CEN supports the identification of changes in the environment that necessitate the inhibition or adaptation of behaviors [95], and CEN activity is implicated in a diverse array of behaviors that includes action planning, sustaining attention, working memory, behavioral inhibition, and cognitive flexibility [96].

DLPFC activity during working memory and cognitive control tasks appears to be a candidate biomarker of pharmacotherapy response. Baseline DLPFC activation during response inhibition that resembled activation in healthy controls differentiated remitters from nonremitters to sertraline, escitalopram, and venlafaxine [97•]. Furthermore, greater DLPFC activity during working memory task was correlated with antidepressant response, but only in patients without childhood maltreatment [98]. Also from the same trial, frontoparietal CEN volume predicted nonremission in a subset of patients [99].

Notably, the DLPFC is also an established neurostimulation target using rTMS, and consequently, its functional correlates have been associated with response to rTMS. High frontostriatal connectivity from the DLPFC [100] has been associated with favorable response to left DLPFC-rTMS. Similar to studies highlighting the relationship between DBS and rTMS targets [74, 94], low baseline connectivity between the DLPFC and sgACC [101••] has also been shown to correlate with antidepressant response to DLPFC-rTMS. Furthermore, electrophysiological DLPFC 
dynamics, including alpha power [102] and entropy [103], have also been linked to antidepressant response to rTMS.

\section{Limbic and Autonomic Circuitry}

Early neuroimaging studies of the limbic system found associations between depression and changes in limbic structures, including decreased hippocampal volume [104], decreased amygdala volume [105], and increased amygdala glucose metabolism [106]. These same changes have also been associated with treatment response; increased hippocampal volume was shown to correlate with improved response to antidepressants [104], increased amygdala volume predicts response to ECT [107] and ketamine [108], and decreased amygdala glucose metabolism correlates with improved response to paroxetine [109]. Overall, limbic structural markers may be seen as an indicator of depression severity, with less severe structural abnormality associated with improved treatment response.

Connectivity between limbic and prefrontal structures has also been shown to be abnormal in MDD in the context of emotional regulation, leading to the hypothesis that decreased top-down control of limbic brain regions by prefrontal input serves as a neural substrate of depressive affect [110]. Recently, fMRI studies have revealed that abnormalities in corticolimbic connectivity in depressed patients are predictive of antidepressant response. Specifically, improved antidepressant response has been correlated with more positive amygdala-vmPFC connectivity [58], more positive amygdala to VLPFC and to ACC connectivity [111], higher amygdala connectivity with right central operculum [112], and lower connectivity with the supplementary motor area and precentral gyrus [112]. These studies support the hypothesis that stronger top-down control of amygdala activity by prefrontal cortices can predict improved treatment outcome.

\section{Internetwork Connectivity as Antidepressant Biomarkers}

It is important to note that the biomarkers reported by many studies do not perfectly align with one IBN, but rather report that connectivity of nodes between many networks is predictive of antidepressant response. For example, tractography studies have reported that sgACC-DBS response correlates with electrode locations that stimulate a distributed network consisting of the amygdala, VMPFC, rACC, dACC, and NAc $[113 \bullet, 114]$. More recently, baseline network characteristics or MDD subtypes based on patterns of whole-brain connectivity have shown promise in predicting antidepressant response to rTMS. We recently showed that functional connectivity-based subtypes of MDD related to anhedonia and anxiety could be used to predict response rates to DMPFC-rTMS [3・•]. Furthermore, whole-brain clustering and network efficiency has been shown to correlate with antidepressant response to accelerated DLPFC-rTMS [115].

\section{Summary}

Baseline differences localized to structural and functional connectivity of the sgACC, DMN, SN, DLPFC, hippocampus, and amygdala predict treatment response (Fig. 1c). However, there does not seem to be a "one-size-fits-all" biomarker for MDD interventions. More specifically, no single biomarker predicts response for all antidepressant interventions or patient populations. Rather, the combined use of complementary biomarkers may allow the comparison of predicted response to different treatments, thereby guiding treatment selection.

At least in adult MDD populations, the most consistently replicated biological marker of antidepressant response is connectivity stemming from the subgenual and rostral ACC. In particular, divergent sgACC connectivity appears to be predictive of antidepressant response to a variety of interventions. sgACC connectivity to nodes of the VMN, SN, and CENthe ventrolateral PFC, insula midbrain, and striatumdifferentially predicts response to $\mathrm{CBT}$ and pharmacotherapy $\left[75 \bullet^{\circ}\right]$. In contrast, connectivity to the ACC and DLPFCnodes of the SN and CEN, respectively - is predictive of response to rTMS $[74,92,101 \bullet \bullet$. Furthermore, structural connectivity from the sgACC to the striatum, ventral PFC, and dorsal PFC is implicated in response to sgACC-DBS [113•].

The $\mathrm{mPFC} / \mathrm{rACC}$ node of the DMN is also implicated in many biomarker studies, including those involving pharmacotherapy [50••], placebo [116], and rTMS [92]. Two large studies from the same consortium report that intact activity of the rostral ACC relative to healthy controls - lower theta power and higher resting-state functional connectivity to the DMN — predicted nonspecific response to three different pharmacotherapies [49, 57]. However, many other studies, albeit without a healthy comparator group, report that higher rACC theta and connectivity to the $\mathrm{SN}$ is predictive of nonspecific antidepressant response, including response to placebo [48•, $50 \bullet, 116]$. Further work is needed to elucidate the relationship between IBN biomarkers of response in comparison to a healthy comparator group.

MDD is also a clinically heterogeneous disorder, and so it is likely that biomarkers attributed to specific MDD subtypes may be required to tailor treatment selection and improve prediction accuracy. To date, one study has identified biological subtypes of MDD that differentially respond to dmPFCrTMS [3••]. In this study, individuals who possessed restingstate functional connectivity abnormalities related to anxiety and insomnia symptom severity responded to rTMS, integrating biologically based MDD subtypes, clinical dimensions, and functional MRI [3••]. Future studies should be mindful 
of the clinical heterogeneity of MDD when identifying biological predictors of response.

\section{Methodological Challenges to Identifying MDD Biomarkers}

\section{Patient Selection}

One area of methodological variability is enrollment criteria. While most studies enrolled adults in a current major depressive episode using DSM-IV or DSM-5 criteria, many identify IBN biomarkers in patients diagnosed with late-life depression [46], adolescent MDD [112], bipolar disorder in a current depressive episode [44], TRD [74], or MDD presenting with other comorbidities [58]. The neurobiological correlates of demographic, diagnostic, and symptom heterogeneity in MDD are not yet fully understood. Consequently, it is possible that the biomarkers generated with modest sample sizes of clinically heterogeneous populations will be challenging to independently replicate, unless investigators take care to replicate the recruitment conditions and diagnostic sample, in addition to the other methods. Identifying biological subtypes based on common abnormal brain function and symptomatology in large trials has been a next logical next step, with our group recently demonstrating that stratifying a heterogeneous group of patients into biologically based subtypes significantly improves prediction accuracy of response to rTMS [3••]. Other examples of using fMRI measures of functional connectivity to parse diagnostic heterogeneity in depression and related affective disorders have also yielded promising results [117-119].

\section{How Do We Define Treatment Response?}

Another critical area of heterogeneity is in each study's definition of treatment response. For example, some studies report linear correlations of neuroimaging measures with the degree of clinical improvement or improvement of a particular symptom. Other biomarkers were generated using categorical predictors of "response," typically $>50 \%$ of a primary outcome measure from baseline to a certain time point into treatment. More challenging, however, is that the therapeutic window that defines "response" for the same intervention varies considerably. For example, escitalopram studies have reported qualitatively different biomarkers based on response at 6 [87], 8 [49], 10 [120], or 12 weeks [68] from intervention onset. Similarly, IBN biomarkers have been identified for CBT response at 12 weeks [68], 14 sessions [73], and 22 sessions [69]. This heterogeneity limits how biomarkers may generalize to independent datasets, and this timeframe need to be clarified in future studies if they are to be translated for wider clinical use. Further longitudinal studies aimed at understanding whether and how the predictors of treatment response at one time point (e.g., 6 weeks) differ from those predicting response at another time point (e.g., 12 weeks).

\section{Considerations for Individual-Level Prediction}

Overfitting is an important consideration in publications that employ machine learning and cross-validation techniques to assess individual-level prediction. Classification accuracy and, therefore, model overfitting is dependent on a number of factors, including the sample size, feature selection, classification methods, and cross-validation [121•]. In one example discussed by Gao and colleagues in their recent review on the machine learning in MDD [121•], leave-one-out crossvalidation improves the classification accuracy of a model because more data is provided during training, but can result in poor model performance in new datasets because the performance of the model is highly dependent on the training dataset. It will be important for future studies to account for the possibility of overfitting by employing methods of feature selection, regression, and classification that limit bias and overfitting [122], as well as machine learning and crossvalidation methods that are less sensitive to the sample size of the training dataset $[121 \bullet]$.

\section{Moving the Field Forward}

Given that less than one third of MDD patients remit on first treatment course [1], prognostic biomarkers of treatment response for specific therapies are in high demand. As outlined in this review, many prognostic biomarkers have been developed for a variety of MDD therapies. However, these neuroimaging biomarkers have yet to be employed in a clinical setting.

A number of practical barriers may prevent neuroimaging biomarkers from being easily measured in the clinical setting, including cost, accessibility, and time to interpretation. For one, many psychiatric clinical settings lack MRI access and on-site expertise [123]. Furthermore, it may not be feasible to schedule, acquire, process, and interpret neuroimaging results, unless these steps can be implemented rapidly. One future possibility might be to collectively concentrate the biomarker search within just one or two MDD subpopulations to minimize the demand on resources while maximizing the potential benefit. TRD biomarkers, for example, might be the most fruitful start, with the aim of identifying the optimal treatment between combination/augmentation pharmacotherapy and more invasive interventions, such as rTMS and ECT, or between different cortical rTMS targets, including the DLPFC and DMPFC.

Moving forward, biomarkers of antidepressant response may be bolstered by the integration of multiple modalities. 
Recent studies integrating structural and functional neuroimaging have shown that the prediction accuracies of integrated models are superior to those trained using single modalities [88]. Furthermore, a new generation of trials is currently underway with the aim of marrying neuroimaging, genetics, and behavioral markers of response [124]. Beyond the brain, trials investigating the pharmacogenetics of drug metabolism are showing promise in furthering our understanding of antidepressant response [125•]. Consequently, it is becoming clear that neuroimaging is certainly a promising "tool in the toolkit" and that its integration with other modalities for response prediction is worthy of future investigation.

\section{Opportunities for Reverse Translation}

The discovery of neuroimaging biomarkers of treatment response in humans offers exciting opportunities for reverse translation to animal models, which allow us to uncover detailed mechanisms of antidepressant action and neural substrates of depression. One potential strategy would use fMRI to identify candidate circuits for optogenetic interrogation in rodent models. Opsins could then be used to recapitulate the abnormal functional connectivity features observed in specific circuits in patients and test whether they are sufficient to drive specific depression-related behaviors. One recent example used optogenetic fMRI in rats to show that reward signals arising from the VTA-to-striatum projection are suppressed by top-down mPFC input and that abnormally elevated functional connectivity between the $\mathrm{mPFC}$ and striatum is sufficient to produce anhedonia [126]. These findings are consistent with a trend seen in a recent fMRI study showing that greater anhedonia-like symptoms in depressed patients may be predicted by a set of functional connectivity changes involving the mPFC, ventral striatum, and other frontostriatal circuits implicated in reward processing, effort valuation, and motivation $[3 \cdot \bullet]$.

Future animal studies may employ similar methods to recapitulate the early connectivity changes highlighted in this review. Such efforts could separate connectivity changes which cause recovery from those which merely correlate with recovery, yielding an avenue for future treatment development. Recent work from our lab has highlighted a large number of connectivity features which are correlated with combinations of symptoms in depressed patients [3••]. Combining optogenetics with fMRI may provide a means to determine which of these connectivity changes are sufficient to drive specific depression-related behaviors. Other methods that can be applied directly to humans, including concurrent TMS/fMRI and network-based analyses of lesion studies, are also promising approaches for understanding how dysfunction in specific circuits contributes to specific behaviors $[127,128]$.

\section{Conclusions}

There is longstanding evidence that brain structure and function is organized into distinct networks of brain connectivity that work in concert to generate complex cognitive patterns and behavior. Research over the past two decades has revealed that abnormal communication within and between these networks underscores the complex psychopathology observed in MDD. Of particular importance to MDD, connectivity of limbic structures, as well as the DMN, SN, CEN, and VMN, give rise to diverse domains of abnormal behavior, including rumination, cognitive control deficits, and anhedonia.

More recent literature provides striking evidence that functional connectivity stemming from networks such as the DMN, $\mathrm{SN}, \mathrm{CEN}$, and VMN can classify treatment responders and nonresponders (Fig. 1c). Most encouragingly, divergent sgACC connectivity appears to be predictive of antidepressant response to a variety of interventions, including pharmacotherapy, psychotherapy, rTMS, and DBS. Studies that employ individual-level prediction have identified neuroimaging biomarkers with the ability to retroactively predict treatment response with over $80 \%$ accuracy, a clinical benchmark of predictive models. However, methodological variables including patient selection, treatment response criteria, and considerations regarding prediction model overfitting will need to need to be addressed in future studies to ensure that these biomarkers are adequate for prospective prediction of treatment response. Prediction accuracy may also be improved by employing models trained on multimodal data.

Nevertheless, identifying generalizable neuroimagingbased biomarkers of treatment response can diminish the suffering of MDD patients by individualizing treatment and accelerating response rates. Biomarkers related to IBN dysfunction may further our understanding of MDD etiology and improve animal models of MDD, thus driving the development of future interventions for this population marked by limited treatment response.

Funding Information Katharine Dunlop was supported by the Canadian Institutes for Health Research Banting Postdoctoral Fellowship. Aleksandr Talishinsky was supported by a Medical Scientist Training Program grant from the National Institute of General Medical Sciences of the National Institutes of Health under award number T32GM07739 to the Weill Cornell/Rockefeller/Sloan Kettering Tri-Institutional MD-PhD Program. Conor Liston was supported by grants from the National Institute of Mental Health, Whitehall Foundation, Hartwell Foundation, One Mind Institute, Rita Allen Foundation, and by a KlingensteinSimons Fellowship in Brain Science.

\section{Compliance with Ethical Standards}

Conflict of Interest Katharine Dunlop and Aleksandr Talishinsky each declare that they have no conflict of interest.

Conor Liston is listed as an inventor for a Cornell University pending patent application, "Neuroimaging Biomarkers for Diagnosing Depression Subtypes." 
Human and Animal Rights and Informed Consent This article does not contain any studies with human or animal subjects performed by any of the authors.

Open Access This article is distributed under the terms of the Creative Commons Attribution 4.0 International License (http:// creativecommons.org/licenses/by/4.0/), which permits unrestricted use, distribution, and reproduction in any medium, provided you give appropriate credit to the original author(s) and the source, provide a link to the Creative Commons license, and indicate if changes were made.

\section{References}

Papers of particular interest, published recently, have been highlighted as:

- Of importance

•- Of major importance

1. Rush AJ, Trivedi MH, Wisniewski SR, et al. Acute and longerterm outcomes in depressed outpatients requiring one or several treatment steps: a STAR*D report. Am J Psychiatry. 2006;163: 1905-17.

2. Trivedi MH, Rush AJ, Wisniewski SR, et al. Evaluation of outcomes with citalopram for depression using measurement-based care in STAR*D: implications for clinical practice. Am J Psychiatry. 2006;163:28-40.

3.• Drysdale AT, Grosenick L, Downar J, et al. Resting-state connectivity biomarkers define neurophysiological subtypes of depression. Nat Med. 2017;23:28-38. A large-scale, multisite study identify biological subtypes of MDD based on patterns of functional connectivity abnormalities that predict response to DMPFC-rTMS.

4. El-Hage W, Leman S, Camus V, Belzung C. Mechanisms of antidepressant resistance. Front Pharmacol. 2013;4:146.

5. Novick D, Montgomery W, Aguado J, Kadziola Z, Peng X, Brugnoli R, et al. Which somatic symptoms are associated with an unfavorable course in Asian patients with major depressive disorder? J Affect Disord. 2013;149:182-8.

6. McTeague LM, Goodkind MS, Etkin A. Transdiagnostic impairment of cognitive control in mental illness. J Psychiatr Res. 2016;83:37-46

7. Fu CHY, Steiner H, Costafreda SG. Predictive neural biomarkers of clinical response in depression: a meta-analysis of functional and structural neuroimaging studies of pharmacological and psychological therapies. Neurobiol Dis. 2013;52:75-83.

8. Sporns O. The human connectome: a complex network. Ann N Y Acad Sci. 2011;1224:109-25.

9. Bullmore E, Sporns O. Complex brain networks: graph theoretical analysis of structural and functional systems. Nat Rev Neurosci. 2009; 10:186-98.

10. Cole MW, Bassett DS, Power JD, Braver TS, Petersen SE. Intrinsic and task-evoked network architectures of the human brain. Neuron. 2014;83:238-51.

11. Laumann TO, Gordon EM, Adeyemo B, et al. Functional system and areal organization of a highly sampled individual human brain. Neuron. 2015;87:657-70.

12. Damoiseaux JS, Rombouts SARB, Barkhof F, Scheltens P, Stam CJ, Smith SM, et al. Consistent resting-state networks across healthy subjects. Proc Natl Acad Sci U S A. 2006;103:13848-53.

13. Greicius MD, Supekar K, Menon V, Dougherty RF. Resting-state functional connectivity reflects structural connectivity in the default mode network. Cereb Cortex. 2009;19:72-8.
14. Hermundstad AM, Bassett DS, Brown KS, et al. Structural foundations of resting-state and task-based functional connectivity in the human brain. Proc Natl Acad Sci U S A. 2013;110:6169-74.

15. Kelly AMC, Uddin LQ, Biswal BB, Castellanos FX, Milham MP. Competition between functional brain networks mediates behavioral variability. Neuroimage. 2008;39:527-37.

16. Koyama MS, Di Martino A, Zuo X-N, Kelly C, Mennes M, Jutagir DR, et al. Resting-state functional connectivity indexes reading competence in children and adults. J Neurosci. 2011;31: 8617-24.

17. Shannon BJ, Raichle ME, Snyder AZ, et al. Premotor functional connectivity predicts impulsivity in juvenile offenders. Proc Natl Acad Sci U S A. 2011;108:11241-5.

18. Vanhaudenhuyse A, Noirhomme Q, Tshibanda LJ-F, et al. Default network connectivity reflects the level of consciousness in noncommunicative brain-damaged patients. Brain. 2010;133:161-71.

19. Power JD, Cohen AL, Nelson SM, et al. Functional network organization of the human brain. Neuron. 2011;72:665-78.

20. Yeo BT, Krienen FM, Sepulcre J, et al. The organization of the human cerebral cortex estimated by intrinsic functional connectivity. J Neurophysiol. 2011;106:1125-65.

21. Dutta A, McKie S, Deakin JFW. Resting state networks in major depressive disorder. Psychiatry Res. 2014;224:139-51.

22. Brakowski J, Spinelli S, Dörig N, Bosch OG, Manoliu A, Holtforth MG, et al. Resting state brain network function in major depression-depression symptomatology, antidepressant treatment effects, future research. J Psychiatr Res. 2017;92:147-59.

23. Shirer WR, Ryali S, Rykhlevskaia E, Menon V, Greicius MD. Decoding subject-driven cognitive states with whole-brain connectivity patterns. Cereb Cortex. 2012;22:158-65.

24. Mulders PC, van Eijndhoven PF, Schene AH, Beckmann CF, Tendolkar I. Resting-state functional connectivity in major depressive disorder: a review. Neurosci Biobehav Rev. 2015;56:330-44.

25. Cowdrey FA, Filippini N, Park RJ, Smith SM, McCabe C. Increased resting state functional connectivity in the default mode network in recovered anorexia nervosa. Hum Brain Mapp. 2014;35:483-91.

26. Sheline YI, Price JL, Yan Z, Mintun MA. Resting-state functional MRI in depression unmasks increased connectivity between networks via the dorsal nexus. Proc Natl Acad Sci U S A. 2010;107: 11020-5.

27. Wagner G, Schachtzabel C, Peikert G, Bär K-J. The neural basis of the abnormal self-referential processing and its impact on cognitive control in depressed patients. Hum Brain Mapp. 2015. https:// doi.org/10.1002/hbm. 22807.

28. Mayberg HS, Liotti M, Brannan SK, et al. Reciprocal limbic-cortical function and negative mood: converging PET findings in depression and normal sadness. Am J Psychiatry. 1999;156:675-82.

29. Philippi CL, Motzkin JC, Pujara MS, Koenigs M. Subclinical depression severity is associated with distinct patterns of functional connectivity for subregions of anterior cingulate cortex. J Psychiatr Res. 2015;71:103-11.

30. Tremblay LK, Naranjo CA, Graham SJ, Herrmann N, Mayberg HS, Hevenor S, et al. Functional neuroanatomical substrates of altered reward processing in major depressive disorder revealed by a dopaminergic probe. Arch Gen Psychiatry. 2005;62:1228-36.

31. Admon R, Nickerson LD, Dillon DG, et al. Dissociable corticostriatal connectivity abnormalities in major depression in response to monetary gains and penalties. Psychol Med. 2015;45:121-31.

32. Manelis A, Almeida JRC, Stiffler R, Lockovich JC, Aslam HA, Phillips ML. Anticipation-related brain connectivity in bipolar and unipolar depression: a graph theory approach. Brain. 2016;139: 2554-66.

33. Harvey P-O, Fossati P, Pochon J-B, Levy R, Lebastard G, Lehéricy $\mathrm{S}$, et al. Cognitive control and brain resources in major 
depression: an fMRI study using the n-back task. Neuroimage. 2005;26:860-9.

34. Holmes AJ, Pizzagalli DA. Response conflict and frontocingulate dysfunction in unmedicated participants with major depression. Neuropsychologia. 2008;46:2904-13.

35. Furman DJ, Hamilton JP, Gotlib IH. Frontostriatal functional connectivity in major depressive disorder. Biol Mood Anxiety Disord. 2011;1:11.

36. Kerestes R, Harrison BJ, Dandash O, Stephanou K, Whittle S, Pujol J, et al. Specific functional connectivity alterations of the dorsal striatum in young people with depression. NeuroImage Clin. 2015;7:266-72.

37. Shulman GL, Fiez JA, Corbetta M, Buckner RL, Miezin FM, Raichle ME, et al. Common blood flow changes across visual tasks: II. Decreases in cerebral cortex. J Cogn Neurosci. 1997;9: 648-63.

38. Dixon ML, Fox KCR, Christoff K. A framework for understanding the relationship between externally and internally directed cognition. Neuropsychologia. 2014;62:321-30.

39. Kucyi A, Davis KD. Dynamic functional connectivity of the default mode network tracks daydreaming. Neuroimage. 2014;100: 471-80.

40. Addis DR, Wong AT, Schacter DL. Remembering the past and imagining the future: common and distinct neural substrates during event construction and elaboration. Neuropsychologia. 2007;45:1363-77.

41. Spreng RN, Mar RA, Kim ASN. The common neural basis of autobiographical memory, prospection, navigation, theory of mind, and the default mode: a quantitative meta-analysis. J Cogn Neurosci. 2009;21:489-510.

42. Maillet D, Rajah MN. Dissociable roles of default-mode regions during episodic encoding. Neuroimage. 2014;89:244-55.

43. Chen CH, Ridler K, Suckling J, Williams S, Fu CHY, Merlo-Pich E, et al. Brain imaging correlates of depressive symptom severity and predictors of symptom improvement after antidepressant treatment. Biol Psychiatry. 2007;62:407-14.

44. Benedetti F, Poletti S, Locatelli C, et al. A Homer 1 gene variant influences brain structure and function, lithium effects on white matter, and antidepressant response in bipolar disorder: a multimodal genetic imaging study. Prog Neuro-Psychopharmacol Biol Psychiatry. 2018;81:88-95.

45. Webb CA, Olson EA, Killgore WDS, Pizzagalli DA, Rauch SL, Rosso IM. Rostral anterior cingulate cortex morphology predicts treatment response to internet-based cognitive behavioral therapy for depression. Biol Psychiatry Cogn Neurosci Neuroimaging. 2018;3:255-62.

46. Gunning FM, Cheng J, Murphy CF, Kanellopoulos D, Acuna J, Hoptman MJ, et al. Anterior cingulate cortical volumes and treatment remission of geriatric depression. Int J Geriatr Psychiatry. 2009;24:829-36.

47. Victoria LW, Alexopoulos GS, Ilieva I, et al. White matter abnormalities predict residual negative self-referential thinking following treatment of late-life depression with escitalopram: a preliminary study. J Affect Disord. 2019;243:62-9.

48. Bartlett EA, DeLorenzo C, Sharma P, et al. Pretreatment and earlytreatment cortical thickness is associated with SSRI treatment response in major depressive disorder. Neuropsychopharmacology. 2018;43:2221-30. A recent example of a large, multisite, placebo-controlled trial identifying structural biomarkers of sertraline response.

49. Arns M, Etkin A, Hegerl U, Williams LM, DeBattista C, Palmer $\mathrm{DM}$, et al. Frontal and rostral anterior cingulate (rACC) theta EEG in depression: implications for treatment outcome? Eur Neuropsychopharmacol. 2015;25:1190-200.

50.• Pizzagalli DA, Webb CA, Dillon DG, et al. Pretreatment rostral anterior cingulate cortex theta activity in relation to symptom improvement in depression: a randomized clinical trial. JAMA Psychiatry. 2018;75:547-54. Another recent example of a large, multisite, placebo-controlled trial identifying functional biomarkers of sertraline response. One of the largest neuroimaging biomarker papers to date.

51. Sämann PG, Höhn D, Chechko N, Kloiber S, Lucae S, Ising M, et al. Prediction of antidepressant treatment response from gray matter volume across diagnostic categories. Eur Neuropsychopharmacol. 2013;23:1503-15.

52. Roffman JL, Witte JM, Tanner AS, et al. Neural predictors of successful brief psychodynamic psychotherapy for persistent depression. Psychother Psychosom. 2014;83:364-70.

53. Spies M, Kraus C, Geissberger N, et al. Default mode network deactivation during emotion processing predicts early antidepressant response. Transl Psychiatry. 2017;7:e1008.

54. van Waarde JA, Scholte HS, van Oudheusden LJB, Verwey B, Denys D, van Wingen GA. A functional MRI marker may predict the outcome of electroconvulsive therapy in severe and treatmentresistant depression. Mol Psychiatry. 2015;20:609-14.

55. Hou Z, Song X, Jiang W, Yue Y, Yin Y, Zhang Y, et al. Prognostic value of imbalanced interhemispheric functional coordination in early therapeutic efficacy in major depressive disorder. Psychiatry Res Neuroimaging. 2016;255:1-8.

56. Andreescu C, Tudorascu DL, Butters MA, Tamburo E, Patel M, Price $\mathrm{J}$, et al. Resting state functional connectivity and treatment response in late-life depression. Psychiatry Res. 2013;214:313-21.

57. Goldstein-Piekarski AN, Staveland BR, Ball TM, Yesavage J, Korgaonkar MS, Williams LM. Intrinsic functional connectivity predicts remission on antidepressants: a randomized controlled trial to identify clinically applicable imaging biomarkers. Transl Psychiatry. 2018;8:57.

58. Philip NS, Barredo J, van't Wout-Frank M, Tyrka AR, Price LH, Carpenter LL. Network mechanisms of clinical response to transcranial magnetic stimulation in posttraumatic stress disorder and major depressive disorder. Biol Psychiatry. 2018;83:263-72.

59. Greicius MD, Flores BH, Menon V, Glover GH, Solvason HB, Kenna H, et al. Resting-state functional connectivity in major depression: abnormally increased contributions from subgenual cingulate cortex and thalamus. Biol Psychiatry. 2007;62:429-37.

60. Drevets WC, Savitz J, Trimble M. The subgenual anterior cingulate cortex in mood disorders. CNS Spectr. 2008;13:663-81.

61. Mayberg HS. Modulating dysfunctional limbic-cortical circuits in depression: towards development of brain-based algorithms for diagnosis and optimised treatment. Br Med Bull. 2003;65:193-207.

62. Mayberg HS, Brannan SK, Mahurin RK, et al. Cingulate function in depression: a potential predictor of treatment response. Neuroreport. 1997;8:1057-61.

63. Mayberg HS, Brannan SK, Tekell JL, Silva JA, Mahurin RK, McGinnis S, et al. Regional metabolic effects of fluoxetine in major depression: serial changes and relationship to clinical response [in process citation]. Biol Psychiatry. 2000;48:830-43.

64. Diekhof EK, Falkai P, Gruber O. Functional neuroimaging of reward processing and decision-making: a review of aberrant motivational and affective processing in addiction and mood disorders. Brain Res Rev. 2008;59:164-84.

65. Dunlop K, Hanlon CA, Downar J. Noninvasive brain stimulation treatments for addiction and major depression. Ann N Y Acad Sci. 2017;1394:31-54.

66. Baeken C, Marinazzo D, Everaert H, Wu G-R, Van Hove C, Audenaert K, et al. The impact of accelerated HF-rTMS on the subgenual anterior cingulate cortex in refractory unipolar major depression: insights from 18FDG PET brain imaging. Brain Stimul. 2015;8(4):808-15.

67. Konarski JZ, Kennedy SH, Segal ZV, Lau MA, Bieling PJ, McIntyre $\mathrm{RS}$, et al. Predictors of nonresponse to cognitive behavioural therapy 
or venlafaxine using glucose metabolism in major depressive disorder. J Psychiatry Neurosci. 2009;34:175-80.

68. McGrath CL, Kelley ME, Dunlop BW, Holtzheimer PE, Craighead WE, Mayberg HS. Pretreatment brain states identify likely nonresponse to standard treatments for depression. Biol Psychiatry. 2014;76:527-35.

69. Sambataro F, Doerig N, Hänggi J, Wolf RC, Brakowski J, Holtforth MG, et al. Anterior cingulate volume predicts response to psychotherapy and functional connectivity with the inferior parietal cortex in major depressive disorder. Eur Neuropsychopharmacol. 2018;28:138-48.

70. Redlich R, Opel N, Grotegerd D, et al. Prediction of individual response to electroconvulsive therapy via machine learning on structural magnetic resonance imaging data. JAMA Psychiatry. 2016;73:557-64.

71. Siegle GJ, Carter CS, Thase ME. Use of FMRI to predict recovery from unipolar depression with cognitive behavior therapy. Am J Psychiatry. 2006;163:735-8.

72. Siegle GJ, Thompson WK, Collier A, Berman SR, Feldmiller J, Thase ME, et al. Toward clinically useful neuroimaging in depression treatment: prognostic utility of subgenual cingulate activity for determining depression outcome in cognitive therapy across studies, scanners, and patient characteristics. Arch Gen Psychiatry. 2012;69:913-24.

73. Rubin-Falcone H, Weber J, Kishon R, Ochsner K, Delaparte L, Doré B, et al. Longitudinal effects of cognitive behavioral therapy for depression on the neural correlates of emotion regulation. Psychiatry Res Neuroimaging. 2018;271:82-90.

74. Salomons TV, Dunlop K, Kennedy SH, Flint A, Geraci J, Giacobbe P, et al. Resting-state cortico-thalamic-striatal connectivity predicts response to dorsomedial prefrontal rTMS in major depressive disorder. Neuropsychopharmacology. 2014;39:488-98.

75.• Dunlop BW, Rajendra JK, Craighead WE, Kelley ME, McGrath CL, Choi KS, et al. Functional connectivity of the subcallosal cingulate cortex and differential outcomes to treatment with cognitive-behavioral therapy or antidepressant medication for major depressive disorder. Am J Psychiatry. 2017;174:533-45. A large study of sgACC functional connectivity biomarkers.

76. Baeken C, Duprat R, Wu G-R, De Raedt R, van Heeringen K. Subgenual anterior cingulate-medial orbitofrontal functional connectivity in medication-resistant major depression: a neurobiological marker for accelerated intermittent theta burst stimulation treatment? Biol Psychiatry Cogn Neurosci Neuroimaging. 2017:2:556-65.

77. Liston C, Chen AC, Zebley BD, Drysdale AT, Gordon R, Leuchter $\mathrm{B}$, et al. Default mode network mechanisms of transcranial magnetic stimulation in depression. Biol Psychiatry. 2014. https://doi. org/10.1016/j.biopsych.2014.01.023.

78. Kozel FA, Rao U, Lu H, Nakonezny PA, Grannemann B, McGregor T, et al. Functional connectivity of brain structures correlates with treatment outcome in major depressive disorder. Front Psychiatry. 2011;2:7.

79. Seeley WW, Menon V, Schatzberg AF, Keller J, Glover GH, Kenna H, et al. Dissociable intrinsic connectivity networks for salience processing and executive control. J Neurosci. 2007;27: 2349-56.

80. Menon V, Uddin LQ. Saliency, switching, attention and control: a network model of insula function. Brain Struct Funct. 2010;214: 655-67.

81. Pleger B, Ruff CC, Blankenburg F, Bestmann S, Wiech K, Stephan KE, et al. Neural coding of tactile decisions in the human prefrontal cortex. J Neurosci. 2006;26:12596-601.

82. Ploran EJ, Nelson SM, Velanova K, Donaldson DI, Petersen SE, Wheeler ME. Evidence accumulation and the moment of recognition: dissociating perceptual recognition processes using fMRI. J Neurosci. 2007;27:11912-24.
83. Lamichhane B, Dhamala M. The salience network and its functional architecture in a perceptual decision: an effective connectivity study. Brain Connect. 2015;5:362-70.

84. Landmann C, Dehaene S, Pappata S, Jobert A, Bottlaender M, Roumenov D, et al. Dynamics of prefrontal and cingulate activity during a reward-based logical deduction task. Cereb Cortex. 2007;17:749-59.

85. Thielscher A, Pessoa L. Neural correlates of perceptual choice and decision making during fear-disgust discrimination. J Neurosci. 2007;27:2908-17.

86. Godlewska BR, Browning M, Norbury R, Igoumenou A, Cowen PJ, Harmer CJ. Predicting treatment response in depression: the role of anterior cingulate cortex. Int J Neuropsychopharmacol. 2018;21:988-96.

87. Godlewska BR, Browning M, Norbury R, Cowen PJ, Harmer CJ. Early changes in emotional processing as a marker of clinical response to SSRI treatment in depression. Transl Psychiatry. 2016;6:e957.

88. Karim HT, Wang M, Andreescu C, Tudorascu D, Butters MA, Karp JF, et al. Acute trajectories of neural activation predict remission to pharmacotherapy in late-life depression. NeuroImage Clin. 2018;19:831-9.

89. Samson AC, Meisenzahl E, Scheuerecker J, Rose E, Schoepf V, Wiesmann $\mathrm{M}$, et al. Brain activation predicts treatment improvement in patients with major depressive disorder. J Psychiatr Res. 2011;45:1214-22.

90. Rizvi SJ, Salomons TV, Konarski JZ, Downar J, Giacobbe P, McIntyre RS, et al. Neural response to emotional stimuli associated with successful antidepressant treatment and behavioral activation. J Affect Disord. 2013;151:573-81.

91. McGrath CL, Kelley ME, Holtzheimer PE, Dunlop BW, Craighead WE, Franco AR, et al. Toward a neuroimaging treatment selection biomarker for major depressive disorder. JAMA Psychiatry. 2013;70:821.

92. Ge R, Blumberger DM, Downar J, Daskalakis ZJ, Dipinto AA, Tham JCW, et al. Abnormal functional connectivity within resting-state networks is related to rTMS-based therapy effects of treatment resistant depression: a pilot study. J Affect Disord. 2017;218:75-81.

93. Bailey NW, Hoy KE, Rogasch NC, Thomson RH, McQueen S, Elliot D, et al. Responders to rTMS for depression show increased fronto-midline theta and theta connectivity compared to non-responders. Brain Stimul. 2018;11:190-203.

94. Fox MD, Liu H, Pascual-Leone A. Identification of reproducible individualized targets for treatment of depression with TMS based on intrinsic connectivity. Neuroimage. 2013;66:151-60.

95. Dosenbach NUF, Fair DA, Cohen AL, Schlaggar BL, Petersen SE. A dual-networks architecture of top-down control. Trends Cogn Sci. 2008;12:99-105.

96. Niendam TA, Laird AR, Ray KL, Dean YM, Glahn DC, Carter CS. Meta-analytic evidence for a superordinate cognitive control network subserving diverse executive functions. Cogn Affect Behav Neurosci. 2012;12:241-68.

97. Gyurak A, Patenaude B, Korgaonkar MS, Grieve SM, Williams LM, Etkin A. Frontoparietal activation during response inhibition predicts remission to antidepressants in patients with major depression. Biol Psychiatry. 2016;79:274-81. One of three recent studies from the iSPOT-D trial identifying biomarkers of response to sertraline, escitalopram, and venlafaxine.

98. Miller S, McTeague LM, Gyurak A, Patenaude B, Williams LM, Grieve SM, et al. Cognition-childhood maltreatment interactions in the prediction of antidepressant outcomes in major depressive disorder patients: results from the iSPOT-D trial. Depress Anxiety. 2015;32:594-604.

99. Korgaonkar MS, Rekshan W, Gordon E, Rush AJ, Williams LM, Blasey C, et al. Magnetic resonance imaging measures of brain 
structure to predict antidepressant treatment outcome in major depressive disorder. EBioMedicine. 2015;2:37-45.

100. Avissar M, Powell F, Ilieva I, Respino M, Gunning FM, Liston C, et al. Functional connectivity of the left DLPFC to striatum predicts treatment response of depression to TMS. Brain Stimul. 2017;10:919-25.

101.• Weigand A, Horn A, Caballero R, Cooke D, Stern AP, Taylor SF, et al. Prospective validation that subgenual connectivity predicts antidepressant efficacy of transcranial magnetic stimulation sites. Biol Psychiatry. 2018;84:28-37. A recent rTMS study to employ prospective validation of a candidate biomarker, an important first step to ensuring that it is generalizable.

102. Woźniak-Kwaśniewska A, Szekely D, Harquel S, Bougerol T, David O. Resting electroencephalographic correlates of the clinical response to repetitive transcranial magnetic stimulation: a preliminary comparison between unipolar and bipolar depression. $\mathrm{J}$ Affect Disord. 2015;183:15-21.

103. Shalbaf R, Brenner C, Pang C, Blumberger DM, Downar J, Daskalakis ZJ, et al. Non-linear entropy analysis in EEG to predict treatment response to repetitive transcranial magnetic stimulation in depression. Front Pharmacol. 2018;9:1188.

104. MacQueen GM, Yucel K, Taylor VH, Macdonald K, Joffe R. Posterior hippocampal volumes are associated with remission rates in patients with major depressive disorder. Biol Psychiatry. 2008;64:880-3.

105. Sheline YI, Gado MH, Price JL. Amygdala core nuclei volumes are decreased in recurrent major depression. Neuroreport. 1998;9: 2023-8.

106. Drevets WC, Price JL, Bardgett ME, Reich T, Todd RD, Raichle ME. Glucose metabolism in the amygdala in depression: relationship to diagnostic subtype and plasma cortisol levels. Pharmacol Biochem Behav. 2002;71:431-47.

107. Ten Doesschate F, van Eijndhoven P, Tendolkar I, van Wingen GA, van Waarde JA. Pre-treatment amygdala volume predicts electroconvulsive therapy response. Front Psychiatry. 2014;5:169.

108. Ortiz R, Niciu MJ, Lukkahati N, Saligan LN, Nugent AC, Luckenbaugh DA, et al. Shank3 as a potential biomarker of antidepressant response to ketamine and its neural correlates in bipolar depression. J Affect Disord. 2015;172:307-11.

109. Saxena S, Brody AL, Ho ML, Zohrabi N, Maidment KM, Baxter LR. Differential brain metabolic predictors of response to paroxetine in obsessive-compulsive disorder versus major depression. Am J Psychiatry. 2003;160:522-32.

110. Rive MM, van Rooijen G, Veltman DJ, Phillips ML, Schene AH, Ruhé HG. Neural correlates of dysfunctional emotion regulation in major depressive disorder. A systematic review of neuroimaging studies. Neurosci Biobehav Rev. 2013;37:2529-53.

111. Vai B, Bulgarelli C, Godlewska BR, Cowen PJ, Benedetti F, Harmer CJ. Fronto-limbic effective connectivity as possible predictor of antidepressant response to SSRI administration. Eur Neuropsychopharmacol. 2016;26:2000-10.

112. Klimes-Dougan B, Westlund Schreiner M, Thai M, GunlicksStoessel M, Reigstad K, Cullen KR. Neural and neuroendocrine predictors of pharmacological treatment response in adolescents with depression: a preliminary study. Prog NeuroPsychopharmacol Biol Psychiatry. 2018;81:194-202.

113. Riva-Posse P, Choi KS, Holtzheimer PE, McIntyre CC, Gross RE, Chaturvedi A, et al. Defining critical white matter pathways mediating successful subcallosal cingulate deep brain stimulation for treatment-resistant depression. Biol Psychiatry. 2014;76:963-9. This is the first study to discuss the association between sgACC-DBS response and structural connectivity of the electrode site.

114. Choi KS, Riva-Posse P, Gross RE, Mayberg HS. Mapping the "depression switch" during intraoperative testing of subcallosal cingulate deep brain stimulation. JAMA Neurol. 2015;72:125260 .

115. Klooster DCW, Franklin SL, Besseling RMH, Jansen JFA, Caeyenberghs K, Duprat R, et al. Focal application of accelerated iTBS results in global changes in graph measures. Hum Brain Mapp. 2019;40:432-50.

116. Sikora M, Heffernan J, Avery ET, Mickey BJ, Zubieta J-K, Peciña M. Salience network functional connectivity predicts placebo effects in major depression. Biol Psychiatry Cogn Neurosci Neuroimaging. 2016;1:68-76.

117. Xia CH, Ma Z, Ciric R, et al. Linked dimensions of psychopathology and connectivity in functional brain networks. Nat Commun. 2018;9:3003.

118. Price RB, Lane S, Gates K, Kraynak TE, Horner MS, Thase ME, et al. Parsing heterogeneity in the brain connectivity of depressed and healthy adults during positive mood. Biol Psychiatry. 2017;81:347-57.

119. Price RB, Gates K, Kraynak TE, Thase ME, Siegle GJ. Datadriven subgroups in depression derived from directed functional connectivity paths at rest. Neuropsychopharmacology. 2017;42: 2623-32.

120. Crane NA, Jenkins LM, Bhaumik R, Dion C, Gowins JR, Mickey BJ, et al. Multidimensional prediction of treatment response to antidepressants with cognitive control and functional MRI. Brain. 2017;140:472-86.

121. Gao S, Calhoun VD, Sui J. Machine learning in major depression: from classification to treatment outcome prediction. CNS Neurosci Ther. 2018;24:1037-52. A recent review article highlighting the methodological challenges of machine learning strategies in MDD.

122. Grosenick L, Shi TC, Gunning FM, Dubin MJ, Downar J, Liston C. Functional and optogenetic approaches to discovering stable subtype-specific circuit mechanisms in depression. bioRxiv 2018;1-33.

123. McLane HC, Berkowitz AL, Patenaude BN, McKenzie ED, Wolper E, Wahlster S, et al. Availability, accessibility, and affordability of neurodiagnostic tests in 37 countries. Neurology. 2015;85:1614-22.

124. Lam RW, Milev R, Rotzinger S, et al. Discovering biomarkers for antidepressant response: protocol from the Canadian biomarker integration network in depression (CAN-BIND) and clinical characteristics of the first patient cohort. BMC Psychiatry. 2016;16: 105.

125. Herbert D, Neves-Pereira M, Baidya R, et al. Genetic testing as a supporting tool in prescribing psychiatric medication: design and protocol of the IMPACT study. J Psychiatr Res. 2018;96:265-72. A protocol paper of a large pharmacogenetics trial to personalize antidepressant treatment.

126. Ferenczi EA, Zalocusky KA, Liston C, et al. Prefrontal cortical regulation of brainwide circuit dynamics and reward-related behavior. Science. 2016;351:aac9698.

127. Chen AC, Oathes DJ, Chang C, Bradley T, Zhou Z-W, Williams $\mathrm{LM}$, et al. Causal interactions between fronto-parietal central executive and default-mode networks in humans. Proc Natl Acad Sci U S A. 2013;110:19944-9.

128. Fox MD. Mapping symptoms to brain networks with the human connectome. N Engl J Med. 2018;379:2237-45.

Publisher's Note Springer Nature remains neutral with regard to jurisdictional claims in published maps and institutional affiliations. 